\title{
Light color changes the density and height of synapses in an area of the brain essential for learning
}

\author{
Levent Tümkaya $^{a}$, Süleyman Kaplan $^{*}$, Ufuk Ö. Mete ${ }^{c}$, Bünyamin Ünal $^{d}$ \\ a Department of Histology and Embryology, Medical Faculty, Rize University, Rize, Turkey \\ ${ }^{b}$ Department of Histology and Embryology, Medical Faculty, Ondokuz Mayis University Samsun, Turkey \\ ${ }^{c}$ Department of Histology and Embryology, Medical Faculty, Çukurova University Adana, Turkey \\ $d$ Department of Histology and Embryology, Medical Faculty, Atatürk University, Erzurum, Turkey
}

\begin{tabular}{|c|c|}
\hline \multicolumn{2}{|c|}{ ARTICLE INFO } \\
\hline \multicolumn{2}{|c|}{ Article History } \\
\hline Received & $27 / 10 / 2010$ \\
\hline Accepted & $27 / 11 / 2010$ \\
\hline \multicolumn{2}{|c|}{ * Correspondence to: } \\
\hline \multicolumn{2}{|c|}{ Süleyman Kaplan } \\
\hline \multicolumn{2}{|c|}{ Department of Histology and Embr } \\
\hline \multicolumn{2}{|c|}{ Medical Faculty, Ondokuz Mayis University } \\
\hline \multicolumn{2}{|c|}{ Samsun, Turkey } \\
\hline e-mail: sk & @omu.edu.tr \\
\hline
\end{tabular}

Key Words :

Learning

Synapses

Asymmetry

Stereology

Wavelength Specificity

Intermediate and Medial Hyperstriatum ventrale

Chick

\section{ABSTRACT}

The effects of pre and post-passive avoidance training, and exposure to different wavelengths of light on numerical synapse density and the mean projected synapse height in the intermediate and medial part of the hyperstriatum ventrale (IMHV) of the chick brain were investigated. Synaptic counts and the mean projected synapse height measurements were done using a 3-dimensional stereological probe, the disector, which requires no assumptions about size, shape, and orientation of synapses. It was found that there was a significant increase in the density of synapses in the left IMHV of chicks in white, yellow and red light illuminations respectively. However, the mean projected height of synapses in these chicks was significantly increased in the right IMHV under white, yellow, green but not red light illumination conditions. These results suggest that numerical and structural modification of synapses may depend on wavelengths of white, yellow, red and green lights.

J. Exp. Clin. Med., 2010; 27:109-113

\section{Introduction}

One of the animal models for learning and memory studies is the use of young chicks which have a mature brain in the early postnatal life. They can learn differences very quickly between food and non-food objects to survive (Stewart et al., 1992). Several forebrain regions of the chick brain, including the hippocampus, lobus parolfactorius and the intermediate and medial hyperstriatum ventrale (IMHV) have been considered to be associated with learning and memory formation (Patterson et al., 1990; Dubbeldam, 1991; Hunter and Stewart, 1993). IMHV of chick forebrain has been demonstrated to be essential for onetrial passive avoidance learning (PAL), which is based on learning to avoid pecking bitter tasting objects (Cherkin, 1969; Patterson et al., 1990). In natural situations this form of learning enables the young chick to avoid pecking at faeces, poisonous substances, insects and grains. The chick is trained by allowing it to peck a bead coated with a bitter-tasting substance, methylanthranilate (MeA), which stimulates an immediate disgust response. Thereafter it will avoid pecking a similar bead when presented at test but it will still peck at other beads of a different color (Rogres, 1996). Controls may be trained on the PAL task by presenting them with a bead coated with water or a dry bead. During the test they will continue to peck the bead. Thus no passive avoidance learning is occurred by continued pecking at any color beads (Rogres, 1996). After PAL, synapse density increases in the IHMV, especially in the left hemisphere. In addition to biochemical (Crove et al., 1994) and morphological studies (Stewart et al., 1992), lesion studies have also shown the dominant role of the left IMHV in learning processes (Patterson et al., 1990; Rose, 1991). Chicks having an IMHV lesion on the first day of hatching trained on day 2 appeared to be amnesic when tested for recall on third day. A similar lesion of the right IMHV fails to impair memory formation (Rose, 1991). Increasing synapse density has been considered to be a morphological change following PAL (Stewart et al., 
1992). A number of studies have suggested that post hatch visual experience can affect the learning ability of chicks. For example, it was found that visual deprivation over the first 3 day-post hatch could produce significant changes in spine density in the hyperstriatum accessorium and in the left IMHV (Galal et al., 1990). Moreover, the effects of pre-hatch light exposure on synaptic development in the IMHV were investigated by Bradley et al., (1994) who found that chicks that hatched in light (but not dark) had a higher synapse density in the left IMHV, whereas the size of synapses in these animals had decreased. It has been suggested that pre and post hatching visual experience may change synaptic characteristics in the IMHV in some way (Bradley et al., 1994). These studies advocate that an increase in synapse density (Hunter and Stewart, 1993; Doubell and Stewart, 1993), synaptic vesicles numbers (Stewart et al., 1984) and the post synaptic length can be expected in the young chicks after learning (Stewart et al., 1991).

The aim of the present study was to investigate whether the color of light in pre and post passive avoidance training has any effect on the numerical synapse density and height of synapses within the IMHV, using unbiased counting methods (Sterio, 1984; Mayhew and Gundersen, 1996; West, 1999).

\section{Materials and methods}

Training: Domestic chicks (Gallus domesticus) were purchased from a commercial hatchery and were brought to the laboratory in the dark cardboard boxes. Many of the studies in which the stereological methods were applied both male and female chicks were used but data were not given separately for sexes (Stewart et al., 1984) or reports did not mention about sexes (Stewart et al., 1991; Doubell and Stewart, 1993; Bradley et al., 1994). To know whether there are any significant differences between the IMHV of sexes, male and female chicks were used in the present study. The chicks were placed in cardboard boxes having $45 \times 25 \times 35 \mathrm{~cm}$ dimensions. The boxes of the animals were separated regarding the light color as "white" (this term was used to describe the broad-spectrum produced by a incandescent bulb), "yellow", "red", and "green" light illuminations. Four different light color conditions were provided by colored incandescent lamps of $60 \mathrm{~W}$. After 24 hours post-hatch pairs of chicks (housed together to reduce the distress often observed in chicks housed singly) were placed in pens one hour before passive avoidance training so that animals could become familiar with the box. Each chick was presented with a white bead five times per 5 minutes. The bead was presented $3-4 \mathrm{~cm}$ from the beak of chick and the response scored as pecked or avoided. Birds that failed to peck the bead in each time were excluded from the study. After 30 minutes, chicks were presented with a similar bead coated with an aversive substance, methylanthranilate, as described by Stewart et al (1984). Chicks pecked this bead once and exhibited a disgust response, with head shaking and wiping their beak on the pens floor. The chicks were tested for avoidance with a similar, dry and white bead both at 1 and $24 \mathrm{hr}$ after training. Birds that give the correct response (avoidance) on training and testing were anaesthetized and perfused.

Perfusion and Fixation: The chicks in each light color illumination group ( $\mathrm{n}=6$ for each), were anaesthetized deeply with ether and then perfused transcardially with $30 \mathrm{ml}$ of $0.9 \%$ saline followed $100-150 \mathrm{ml}$ of $2 \%$ paraformaldehyde/glutaraldehyde in $0.1 \mathrm{M}$ phosphate buffer. Both saline and fixative were preheated to $37^{\circ} \mathrm{C}$ before used. The brains were then removed and the right and left IMHV dissected out in according to Horn (Horn et al., 1985; Horn, 1991). The tissues were stored in the same fixative overnight and prepared for electron microscopy by post fixing in \% 1 osmium tetroxide for one hour, dehydrating through graded alcohols, and embedding in Araldite CY-212 resin kit. Blocks were hardened in an oven at $65-70^{\circ} \mathrm{C}$ for $48 \mathrm{hr}$. All subsequent procedures were performed 'blind' by given random numbers to the tissue blocks.

Microscopy: One block (each approximately 1 $\mathrm{mm}^{3}$ ) from each hemisphere was used to estimate synapse density. Ultrathin sections of silver interference color were cut by an ultramicrotome and mounted on grids. Five grids of ultrathin sections were taken from each block. They were stained with uranyl acetate and lead citrate. A grid had a ribbon of serial sections and ribbons on successive grids were separated by at least $2 \mu \mathrm{m}$ so that samplings would not be performed in adjacent regions. The sections were examined via a Zeiss EM 900 electron microscope operated at an accelerating voltage of $60 \mathrm{kV}$. Estimation of the numerical synapse density and the mean height of synapse were done using the disector counting method (Sterio, 1984). In this method a pair of adjacent sections was used one of which was the reference and other was the look-up section. From each grid a pair of micrographs was taken at a magnification of 5000x of the equivalent area in the adjacent sections. Final magnification of electron micrographs was 27000 . Two unbiased counting frames were superimposed on each pair of micrographs so that the same area of adjacent micrographs, i.e. the adjacent sections, could be easily found. The bottom and left boundaries of the frame were considered to be the forbidden lines (Gundersen, 1977). Any synapse crossing these lines was not included for the analysis. Synapses were counted if they were present in the reference section but not in the look-up section (Sterio, 1984). If a post-synaptic density was associated with at least three synaptic vesicles, this was considered as a synapse. Because the major synapse population is asymmetric, and symmetric synapses were too low to provide a representative sample, the values for these synapses were not given separately. Therefore, statistical analysis of electron micrographs from the study is presented here for both types of synapses. Section thickness was estimated according to the Small's 'fold' technique. For this method, each section was scanned to find a minimal fold, and then photographed. The smallest 
visible width of the fold, which is twice the section thickness, was measured. Reliability of this method has been shown to be as accurate as any other means of estimating section thickness (DeGroot, 1988).

The mean numerical synapse density per $\mathrm{mm}^{3}$ $\left(\mathrm{N}_{\mathrm{v} \text { syn }}\right)$ was estimated using the following formula;

$$
\mathbf{N}_{\mathrm{v} \text { syn }}=\Sigma \mathbf{Q}^{-} / \mathbf{V}_{\text {dis }}
$$

Where $\Sigma \mathrm{Q}^{-}$is the total number of counted synapse profiles, which are called disector particle that appears only in the reference section but not in the look-up section, $\mathrm{V}_{\text {dis }}$ is the disector volume.

The mean projected synapse height $\left(\mathrm{H}_{\text {syn }}\right)$, a measure of the size of postsynaptic density, was estimated from following formula;

$$
\mathbf{H}_{\mathrm{syn}}=\left(\Sigma \mathbf{Q}_{\mathrm{syn}} / \Sigma \mathbf{Q}^{-}\right) \cdot \mathbf{t}
$$

Where $\Sigma \mathrm{Q}_{\mathrm{syn}}$ is the total number of synapses in the unbiased counting frame on reference section and $t$ is the thickness of reference section. To increase reliability of the study counting more synapse is advisable. For this reason the same pair of plains, i.e. micrographs, was used in both forward (reference to look-up) and reverse (look-up to reference) directions and the average of these two counts was taken. Reliability of both $\left(\mathrm{N}_{\mathrm{v} \text { syn }}\right)$ and $\left(\mathrm{H}_{\text {syn }}\right)$ estimations (the coefficient of error) were determined (16). Estimations were considered to be relevant if the coefficient of error is approximately $10 \%$. A total of 1457 synapses were used to estimate of $\left(\mathrm{N}_{\mathrm{vsyn}}\right)$ and $\left(\mathrm{H}_{\mathrm{syn}}\right)$ from 400 disector pairs. All data were analysed using an analysis of variance (ANOVA) to examine the differences between the right and left mean synaptic density values and projected mean synapse height in different light color conditions. Differences were considered to be significant at values of $\mathrm{P}<0.05$.

All experiments were carried out according to the guidelines of the European Community Council for experimental animal care.

\section{Results}

According to our observations, chicks in the white light box were more active displayed as having more pecking reflex than in the other color illuminations. This difference was also noticed during the training task. Following passive avoidance training, numerical synapse density $\left(\mathrm{N}_{\mathrm{v} \text { syn }}\right)$ and the mean projected synaptic height $\left(\mathrm{H}_{\text {syn }}\right)$ in the IMHV were shown to be affected by the light color. However when the same hemisphere was compared between male and female chicks (i.e. left vs. left or right vs. right) there were no significant differences in numerical synapse density in the IMHV for a given illumination color. In other words, there was no dimorphism in IMHV between sexes, which were exposed to the same light color. In many of the stereological studies dealing with synapse density and synapse height in the chick IMHV were used both sexes and their comparisons were between the left and right side of IMHVs but not between sexes (Bradley et al., 1994; Doubell and Stewart, 1993; Stewart et al., 1984;
Mayhew and Gundersen, 1996). Therefore $\mathrm{N}_{\mathrm{v} \text { syn }}$ values of the corresponding hemispheres of male and female chicks were summed, and the average mean of $\mathrm{N}_{\mathrm{V} \text { syn }}$ was taken for both sexes. Following training in all groups of birds except the chicks in green light $(\mathrm{F} 1,46=2.55, \mathrm{P}>0.05)$, there was a significant difference in between numerical synapse density between the right and left IMHV (values were $\mathrm{F} 1,46=11.83, \mathrm{P}<0.001$ in white, $\mathrm{F} 1,46=4.66$, $\mathrm{P}<0.05$ in yellow and $\mathrm{F} 1,46=6.80, \mathrm{P}<0.05$ in red light illuminations). The left IMHV has noticeably higher $\mathrm{N}_{\mathrm{v}}$ than the right IMHV in the white, yellow and red light illuminations (Fig 1).

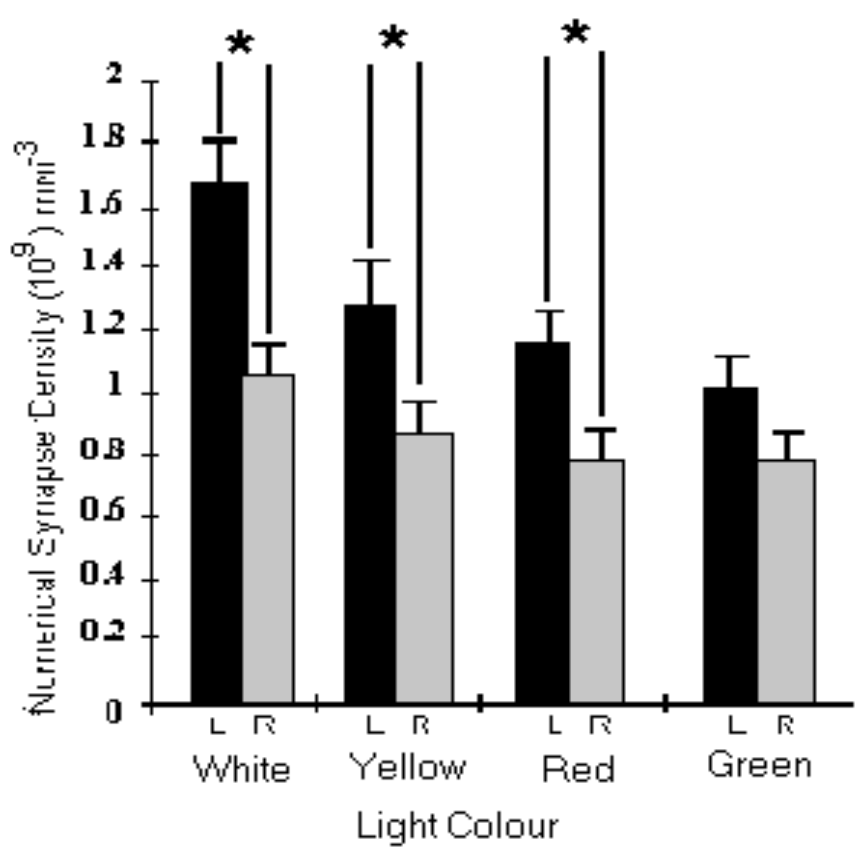

Fig 1. Mean numerical synapse density \pm SEM of the left $(\mathrm{L})$ and right $(\mathrm{R})$ IMHV, from white $(\mathrm{W})$, yellow $(\mathrm{Y})$, red $(\mathrm{R})$ and green $(\mathrm{G})$ light illumination, at 24 hours following passive avoidance training. Each estimate is based on results obtain from 6 chicks. ${ }^{*} \mathrm{P}<0.05$

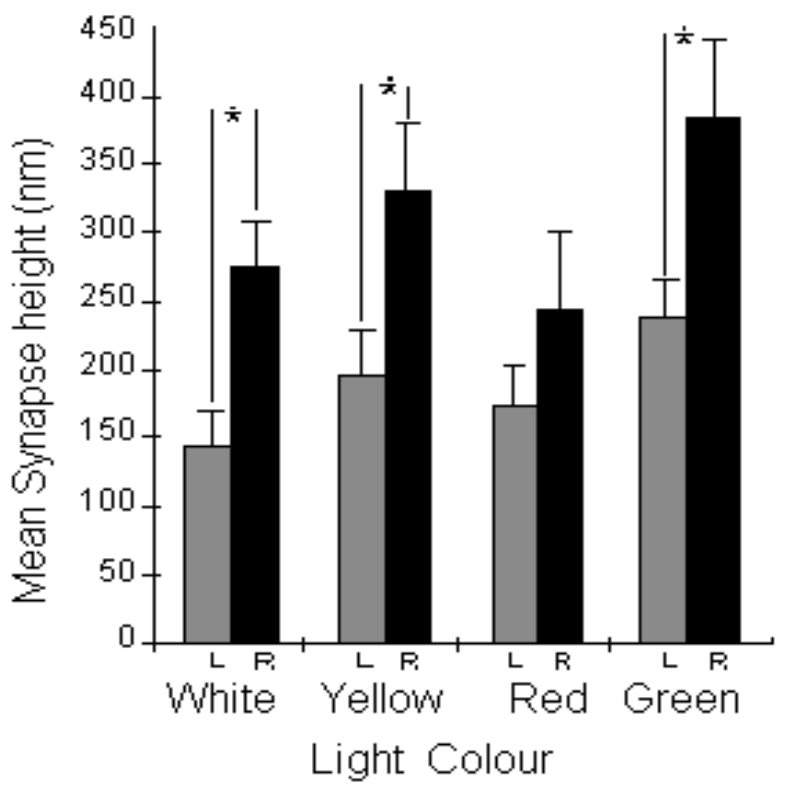

Fig 2. Mean projected height of synapse \pm SEM of the left (L) and right (R) IMHV from white $(W)$, yellow $(Y)$, red $(R)$ and green $(G)$ light illumination, at 24 hours following passive avoidance training. $* \mathrm{P}<0.05$ 
The mean projected synapse height was not significantly different between the same side of the IMHV of male and female chicks in the same light color illumination except in white light in which it is difficult to interpret why the difference between male and female right IMHV's has rised to a significant degree in contrast to other illumination colors. Thus, total $\mathrm{H}_{\mathrm{syn}}$ of the male and female chicks was divided to take an average of $\mathrm{H}_{\text {syn }}$ for comparison of the left and right hemispheres. Except in red light $(\mathrm{F} 1,46=1.98, \mathrm{P}>0.05)$, there was a significant difference between the mean projected synapse height of right and left IMHV of both sexes in the same light color illumination (Fig 2). In all groups (in white, F1,46 = 5.54, $\mathrm{P}<0.05$, in yellow $\mathrm{F} 1,46=4.45, \mathrm{P}<0.05$, in green $\mathrm{F} 1,46$ $=4.59, \mathrm{P}<0.05)$ but not in red light color, Hsyn of the right IMHV was significantly higher than in the left.

\section{Discussion}

The main findings of the present study are as follows: Twenty four hours after one-trial passive avoidance training of day-old chicks in different light color conditions as white, yellow and red, there is a significant increase in $\mathrm{N}_{\mathrm{v} \text { syn }}$, in the left IMHV compared to the right hemisphere except the chicks under green light. However in the white, yellow and green lights (but not in red light) $\mathrm{H}_{\text {syn }}$ is significantly higher in the right than in the left IMHV.

The results presented here are based upon the numerical density of synapses in the IMHV. Although those alterations in numerical synaptic density are significant, it should be guaranteed that the reference volume (i.e. volume of the IMHV) was not difference between groups. Clearly an increase or a decrease in $\mathrm{N}_{\mathrm{V} \text { syn }}$ may not reflect an increase or a decrease in number of synapses if the reference volume decreases or increases by a relevant amount. It was reported that after $24 \mathrm{hr}$ passive avoidance training no changes occurred in the volume of the lobus parolfactorius (LPO), a region of chick forebrain Hunter (1991) cited by Doubell and Stewart (1993). One day after training, a similar result can be expected for the IMHV (Doubell and Stewart, 1993).

Patel and Stewart have shown an importance of morphological changes in the left hemisphere in visual discrimination learning (Patel and Stewart, 1988). In their study, the number and structure of dendritic spines were investigated one day after passive avoidance training and it has been found that the increase of the spine density is more pronounced in the left IMHV. It is likely that changes in spine shape and an increase of spine density observed in the left hemisphere may be involved in modulating synaptic connectivity, since information reaches the cell body via dendritic spines (Patel and Stewart, 1988) Gaston and Gaston have also suggested that memory for complex pattern discrimination task may be stored only in the left hemisphere (Gasten and Gasten, 1984). Moreover, the pathways used in the passive avoidance learning task are located in the left hemisphere, and interfering with this hemisphere disrupts their development, but similar disruption is not observed following similar treatment in the right hemisphere (Rogers, 1986).

Visual discrimination learning, of which the passive avoidance learning is an example, is predominantly a left hemisphere function. Studies, which have tested the visual discrimination in the chick, have consistently shown the importance of the right eye and the left hemisphere pathway in acquisition of such tasks (Rogers, 1986). The results presented here in which the left hemisphere has a superiority to the right hemisphere in terms of increase in $\mathrm{N}_{\mathrm{V} \text { syn }}$ after training in certain illumination colors are consistent with the idea of the importance of the left hemisphere in visual discrimination learning (Gasten and Gasten, 1984; Rogers, 1986; Patel and Stewart, 1988; Doubell and Stewart, 1993).

Another major finding in the present study is that the difference in the mean projected synapse height between the right and left IMHV was significantly higher in the right IMHV than the left in white, yellow and green but not in red light illuminations. This may have a close correlation with the increase in the numerical synapse density. An increase in numerical synapse density in one side of IMHV would be expected to be compensated by a decrease in the mean projected synapse height in the same side. This may result from the preferential addition of synapses and it has also been postulated that the new synapses which are added as a result of exposure to the white, yellow, and green lights in the left IMHV will be less mature and smaller in size than existing synapses. Following an increase of $\mathrm{N}_{\mathrm{Vsyn}}$ a decrease in $\mathrm{H}_{\text {syn }}$ could result from splitting of pre-existing synapses (Calverley and Jones, 1990); however, our data are not consistent with this idea. Since no significant difference was found between the $\mathrm{N}_{\mathrm{Vsyn}}$ of the left and right IMHV in the green light illumination, $\mathrm{H}_{\text {syn }}$ of the right IMHV was significantly higher than the left IMHV under green light. On the other hand the left $\mathrm{N}_{\mathrm{vsyn}}$ increased significantly, whereas the expected significant increase of the right $\mathrm{H}_{\text {syn }}$ was not observed in red light illumination. These results suggest that increasing of numerical synapse density in the IMHV may result from addition of new-formed synapse, but not splitting of pre-existing synapses. This idea would be correct if there were no significant differences between $\mathrm{H}_{\text {syn }}$ of the right and left in any light color condition. In fact, this occurred in red light illumination where $\mathrm{N}_{\mathrm{Vsyn}}$ of the left IMHV was significantly higher than in the right IMHV. Eggs that were exposed to light and dark rear that is three days pre hatching and 6 hours post hatching. After that $\mathrm{N}_{\mathrm{v} \text { syn }}$ of IMHV of the light and dark hatched chicks was compared (Bradley et al., 1994). $\mathrm{N}_{\mathrm{Vsyn}}$ of the left IMHV of the light reared chicks was found higher than in the left and right IMHV of dark reared chicks. The results of the present study are consistent with a previous study (Bradley et al., 1994), and show an obvious effect of different wavelengths provided before and after passive avoidance learning.

Our findings support an idea that after passive avoidance learning task numerical synapse density 
increases significantly in the left but not in the right IMHV. The present study also demonstrates that synaptic density changes after passive avoidance learning in the chick are wavelength specific.

Prof. Dr. Michael G. Stewart (Open University in UK.) and Prof. Dr. Beytullah Afsin are sincerely thanked for excellent reviewing. We would also like to thank Prof. Dr. Mehmet Kaya, Ms. Gunseli Tuygun and Mr. Nevruz Dural for providing laboratory facilities and assistance; Ondokuz Mayıs University Research Fund supported (T144) this work.

\section{REFERENCES}

Bradley, P.M., Burns, B.D., Kaplan, S., Webb, A.C. 1994. Effects of light hatching on synapse number and size in the intermediate and medial part of the hyperstriatum ventrale of the domestic chick. Dev. Brain Res. 80, 295-298.

Calverley, R.K.S., Jones, D.G. 1990. Contributions of dendritic spines and perforated synapses to synaptic plasticity. Brain Res. Rev. 15, 215-249.

Cherkin, A.,1969. Kinetics of memory consolidation: role of amnesic treatment parameters. Proc. Natl. Acad. Sci. 63, 1094-1101.

Crowe, S.F., Zhao, W-Q, Sedman, G.L., 1994. 2-Deoxygalactose interferes with an intermediate processing stage of memory. Behav. Neural. Bio. 61, 206-213.

DeGroot, D.M.G.1988. Comparison of the methods for the estimation of the thickness of ultra thin tissue sections. J. Microsc. 151, 23-42.

Dubbeldam, J.L. 1991. The avian and mammalian forebrain correspondences and differences. Neuronal.behav. plasticity. New York: Oxford University Press. 67-91.

Doubell, T.P., Stewart, M.G. 1993. Short-term changes in the numerical density of synapses in the intermediate and medial hyperstriatum ventrale following one-trial passive avoidance training in the chick. J. Neurosci.13, 230-2236

Galal, K.M., Bradley, P.M., Drummond, P. 1990. The effect of dark rearing on dendritic development in two regions of the forebrain of the chick. Dev. Brain Res. 53,135-138.

Gasten, K.E., Gasten, M.G. 1984. Unilateral memory after binocular discrimination training: left hemisphere dominance in the chick. Brain Res. 303, 190-193.

Gundersen, H.J.G. 1977. Notes on the estimation of the numerical density of arbitrary profiles: the edge effect. J. Microsc. 111, 219-223.

Horn, G. 1991. Technique for removing IMHV from the chick brain, and Imprinting and recognition memory; a review of neural mechanisms. Neural. Behav. Plasticity. New York: Oxford Univ Press.44-48 and 219-261.

Horn, G., Bradley, P., McCabe, M.J. 1985. Changes in the structure of synapses associated with learning. J. Neurosci. 5, 3161-3168.

Hunter,A., Stewart, M.G. 1993. Long-term increases in the numerical density of synapses in the chick lobus parolfactorius after passive avoidance training. Brain Res. 605, 251-255.

Hunter, A. 1991. Synaptic plasticity in the chick lobus parolfactorius during development and following memory formation. PhD thesis, The Open University UK.

Mayhew, T.M., Gundersen, H.J.G. 1996. 'If you assume, you can make an ass out of and me': a decade of disector for stereological counting of particles in space. J. Anat. 188, 1-15.

Patterson, T.A., Gilbert, D.B., Rose, S.P.R. 1990. Pre- and post-training lesions of the intermediate medial hyperstriatum ventrale and passive avoidance learning in the chick. Exp. Brain Res. 80,189-195.

Patel, S.N., Stewart, M.G. 1988. Changes in the number and structure of dentritic spines 25 hours after passive avoidance training in the domestic chick. Brain Res. 449, 34-46.

Rogres, L.J. 1996. Behavioral structural and neurochemical asymmetries in the avain brain: a model system for studing visual development and processing. Neurosci. Biobehav. Rev. 20, 487-503.

Rose, S.P.R. 1991. How chicks make memories: the cellular cascade from C-fos to dentritic remodeling. Trends Neurosci. 14, 390-397.

Rogers, L.J. 1986. Lateralizastion of learning in chicks. Adv. Study Behav. 16, 147-189.

Stewart, M.G., Rose, S.P.R., King, T.S., Gabbott, P.L.A., Bourne, R. 1984. Hemisphere asymmetry of synapses in chick medial hyperstriatum ventrale following passive avoidance training: a stereological study. Dev. Brain Res. 12, 261269.

Stewart, M.G. 1991. Changes in dentritic and synaptic structure in chick forebrain consequent on passive avoidance learning. Neural. behavioral plasticity. New York: Oxford Univ Press; 305-328.

Stewart, M.G., Lowdnes, M., Hunter, A. 1992. Memory storage in chicks involves an increase in dendritic spine number and synaptic density. Brain Dysfunction 5, 50-64.

Sterio, D.C. 1984. The unbiased estimation of number and sizes of arbitrary particles using the disector. J. Microsc. 134, 127-136.

West, M.J. 1999. Stereological methods for estimating the total number of neurons and synapses: issues of precision and bias. Trends Neurosci. 22, 51-61. 